\title{
A RESOLUBILIDADE DA ASSISTÊNCIA COMO PROPOSTA DE INDICADOR PARA AVALIAR A DESCENTRALIZAÇÃO DA ASSISTÊNCIA HOSPITALAR EM MINAS GERAIS ${ }^{1}$
}

\section{AN INDICATOR FOR ASSESSING THE DECENTRALIZATION OF HOSPITAL CARE IN MINAS GERAIS}

\section{UN INDICADOR PARA LA EVALUACIÓN DE LA DESCENTRALIZACIÓN DE LA ATENCIÓN HOSPITALARIA EN MINAS GERAIS}

\author{
André Amorim \\ Secretaria de Estado da Saúde de Minas Gerais \\ amorim.andrel@gmail.com
}

\begin{abstract}
RESUMO
Este artigo apresenta o indicador denominado Resolubilidade da Assistência Hospitalar. Fundamentalmente, tal indicador mensura o percentual de internações realizado nas unidades de referência, em relação ao total de internações para os munícipes de cada município ou região de saúde, por nível de atenção à saúde. O trabalho busca contribuir com a leitura do desempenho do parque hospitalar do Estado e fornecer instrumental para avaliação da descentralização de tais serviços. O detalhamento da metodologia de construção de tal indicador será também abordado, incluindo a sua concepção, etapas de cálculo e validação. A análise do indicador de Resolubilidade apontou que, na última década, houve expansão de parcela considerável de serviços hospitalares de alto grau de complexidade tecnológica em Minas Gerais, derivada da política de descentralização engendrada pelo Estado, apesar da manutenção das disparidades regionais.
\end{abstract}

Palavras-Chaves: Políticas Públicas de Saúde. Descentralização. Escala

\section{ABSTRACT}

This paper presents an indicator of Health Care. Mainly, this indicator measures the percentage of admissions made in reference units, relative to the total number of hospitalizations for residents of each municipality or health region, by level of health care. The work seeks to contribute to the reading performance of the state park hospital and provide instrumental assessment of decentralization of such services. The details of the methodology for the construction of this indicator will also be discussed, including its design, calculation and validation steps. The analysis of the indicator pointed out that in the last decade, there has been considerable expansion portion of hospital services high degree of technological complexity in Minas Gerais, because of the decentralization policy engendered by the state, despite the maintenance of regional inequalities.

Key-words: Public Policy Health Decentralization. Scale

\section{RESUMEN}

En este trabajo se presenta un indicador para la atención hospitalaria. Fundamentalmente, este indicador mide el porcentaje de los ingresos realizados en unidades de referencia, en relación con el número total de hospitalizaciones por los residentes de cada municipio o región de salud, según el nivel de atención de la salud. El trabajo busca contribuir a la capacidad de lectura del hospital parque estatal y proporcionar una evaluación instrumental de la descentralización de los servicios. También se discuten los detalles de la metodología para la construcción de este indicador, incluyendo su diseño, cálculo y pasos de validación. El análisis del indicador de solvencia señaló que en la última década, ha sido parte de expansión considerable de los servicios hospitalarios

\footnotetext{
${ }^{1}$ Submetido em 20 de Agosto de 2013. Aceito em 27 de Setembro de 2013. O artigo foi avaliado segundo o processo de duplo anonimato além de ser avaliado pelo editor. Editores responsáveis: Márcio Augusto Gonçalves e Lucas Maia dos Santos. Reprodução parcial ou total e trabalhos derivativos permitidos com a citação apropriada da fonte.
} 
de alto grado de complejidad tecnológica en Minas Gerais, derivado de la política de descentralización, engendrado por el Estado, a pesar del mantenimiento de las disparidades regionales.

Key Words: Salud Pública Descentralización Política. escala

\section{INTRODUÇÃO}

A avaliação de políticas públicas é um tema vasto para a pesquisa científica, seja ela aplicada ou não. O tema, pelo seu amplo conteúdo teórico, aliado a um componente significativo de análise da realidade concreta, desperta um interesse eminente tanto de estudos acadêmicos - em seu sentido mais estrito -, como de trabalhos de uma linha mais mercadológica (consultorias para órgãos governamentais, por exemplo).

O campo da saúde pública mostra-se como bom exemplo para essa assertiva. Em primeiro lugar, pela responsabilidade de se lidar com as condições do bem-estar dos indivíduos - em última instância, desperdiçar recursos públicos da saúde pública é desperdiçar anos saudáveis de vida. Em segundo lugar, pela singularidade envolvida no emaranhado de fatores que contribuem para uma prestação de serviços públicos de saúde - a chamada multicausalidade - sejam eles fatores endógenos ou exógenos, o que complexifica ainda mais as análises. Zucchi et. al. (2000), Galvarro et. al. (2012), dentre outros autores, discorreram sobre os elementos que podem influenciar no acesso da população aos serviços de saúdei.

Em terceiro lugar, releva-se o debate a respeito do financiamento. Num plano macro, têm-se as campanhas atuais para disponibilização de mais recursos para o orçamento da seguridade social, essencialmente por parte da União ${ }^{i i}$. Num plano micro, ressalvam-se as preocupações: i) da redução do custo de oportunidade e da desconstrução do gasto futuro em saúde, ambas afetas ao campo da Economia da Saúde, típicas dos agentes financiadores; ii) de se adequar as relações custo-benefício e custo-efetividade, típicas dos gerentes dos prestadores de serviços.

Assim, é de se esperar que pesquisadores e gestores estejam aptos a lançar luz sobre o binômio gestão e financiamento, numa busca constante pela garantia do acesso - qualificado, equânime e oportuno - de cada usuário ao sistema de saúde, sem perder de vista a sustentabilidade financeira. Desafio hercúleo para quem se dispõe a fazer parte do maior sistema universal de saúde pública do mundo - o Sistema Único de Saúde Brasileiro (SUS).

Nessa teia, releva-se a necessidade de se estabelecer medidas de monitoramento e avaliação, isentas e refutáveis, que permitam a comparabilidade do desempenho de gestores e prestadores de saúde ${ }^{\mathrm{iii}}$. Especialmente sobre o desempenho das unidades hospitalares.

La Forgia e Couttolenc (2008) ressalvam que, no Brasil, as despesas com hospitais representam cerca de setenta por cento do gasto total com saúde. Além disso, frisam também que o SUS é a principal fonte de financiamento para a atenção hospitalar, correspondendo a uma média de $60 \%$ das divisas para esses prestadores. Finalmente, em que pesem as diretrizes legais do SUS sustentarem a ordenação do acesso do usuário a partir do nível de complexidade mais básica - o da atenção primária -, renomados autores (LA FORGIA E COUTTOLENC, 2008, MENDES E PESTANA, 2004) ainda alertam sobre nossa cultura hospitalocêntrica.

Partindo dessa questão, este artigo se propõe a apresentar e discutir uma proposta de avaliação da assistência hospitalar em Minas Gerais, tomando como premissa que a regionalização foi a estratégia de descentralização de serviços adotada pelo SUS. Tem como objetivo geral: avaliar a evolução da descentralização dos serviços hospitalares em Minas Gerais; e específicos: 
(i) apresentar o indicador de resolubilidade da assistência hospitalar, detalhando sua metodologia de concepção e cálculo, a partir da validação da Tipologia ou Carteia de Serviços Hospitalares por um painel de especialistas;

(ii) Demonstrar como o princípio da economia de escala se desdobra em diretrizes operacionais que orientam a descentralização de serviços de saúde em Minas Gerais e a sua posterior avaliação.

Trata-se de uma pesquisa exploratória. Foram utilizadas a análise bibliográfica, análise documental e análise quantitativa de dados secundários. Os dados secundários foram obtidos dos sistemas oficiais de informações do Sistema Único de Saúde (DATASUS). Foi investigado o estado de Minas Gerais, a partir dos seus municípios e regiões de saúde, desde o ano de 2003 até o ano de 2011.

\section{REFERENCIAL TEÓRICO}

Através da Teoria Contingencial, tem-se que as empresas se modificam internamente para manter vantagens competitivas sustentáveis, pois o ambiente é mandatário para o processo decisório. Nesse sentido, imperioso abordar as dimensões às quais se sujeitam as unidades hospitalares em análise. Portanto, esta seção dispõe duas das principais macrodimensões sobre as quais se assentam o arcabouço causal de estratégias a serem tomadas por cada instituição hospitalar: o modelo de gestão (das unidades e do sistema); e o modelo de descentralização adotado no estado de Minas Gerais.

\subsection{Implicações dos modelos de gestão na contratualização dos hospitais}

Desde a fundação das mais tradicionais Casas de Caridade ou Santas Casas, passando pelos hospitais erguidos na época da ditadura militar, até os modelos contemporâneos de parcerias público-privadas, há hoje, no Brasil, diversas formas de vínculos dessas unidades prestadoras. Tal cenário é o que se observa, também, no Estado de Minas Gerais.

De acordo com o Cadastro Nacional de Estabelecimentos de Saúde (CNES), há 699 (seiscentos e noventa e nove) unidades hospitalares em Minas Gerais, das quais: 324 entidades filantrópicas; 216 empresas privadas; 99 pertencem à Administração; 35 têm outro vínculo público (autarquia, fundação ou empresa); e as 25 restantes se distribuem por mais outros 4 tipos distintos de natureza administrativa.

As organizações burocráticas visam reduzir a incerteza e o oportunismo que o mecanismo regulador "preço" do mercado não consegue exaurir por si só (OUCHI, 1980). No entanto, aquela diversidade de vínculos tende a reduzir a eficiência esperada nos mecanismos de contratualização entre as unidades hospitalares e os entes federados que concorrem para seu financiamento, na medida em que a expectativa das partes terá um amplo espectro de gradações possíveis (administração direta, entidade filantrópica, empresa privada, cada qual com sua composição, plano de negócio, estratégia).

Nos hospitais sob administração direta, por exemplo, os mecanismos de responsabilização local podem ser minimizados, na medida em que seu orçamento centralizado prejudica a autonomia decisória (da diretoria clínica e administrativa).

Nessa vertente, La Forgia e Couttolenc (2008, op. cit) alertam que a descentralização dos serviços de saúde, no Brasil, se concentrou no fortalecimento do poder dos governos locais e não no fortalecimento de gestores de programas ou unidades de saúde. Nesse sentido, asseveram que

Na maioria dos casos, a autoridade decisória foi centralizada nas secretarias de saúde estaduais ou municipais ou em outras instâncias da administração 
pública. A reforma [sanitária] alterou pouco a maneira como a maioria dos hospitais públicos são governados ou gerenciados, ou como os hospitais privados no âmbito do SUS são contratados e monitorados (LA FORGIA E COUTTOLENC, 2008, p. 21).

Além disso, partindo da tipologia de Ouchi (1980), tem-se, pelo menos, duas organizações coabitando o mesmo local físico do hospital: a organização hierárquica e a organização "clâ". Caso ocorra uma divergência de objetivos entre essas organizações (numa propositura de metas de produtividade, por exemplo), cada qual tende a maximizar sua utilidade. Tal situação pode prejudicar a credibilidade e a confiança entre o hospital e os seus entes financiadores ${ }^{\text {iv }}$.

Sendo assim, há, portanto, uma necessidade de se criar uma percepção de equidade nas entregas de ambas as partes (serviços hospitalares prestados a contento e financiamento adequado), que, no caso de dificuldade em determinar o valor de tais bens ou serviços, tendese a onerar os custos de transação entre prestadores e financiadores. Qual seria a medida adequada para a remuneração de cada unidade? De cada profissional dessa unidade? Quais os mecanismos a serem empregados para realizar essa aferição?

Ao passo que, no mercado, o mecanismo regulador é o preço, na burocracia, tem-se as regras (OUCHI, 1980, op. cit). Dessa feita, em Minas Gerais, a política hospitalar visa remunerar melhor aquelas unidades que tenham um atendimento proporcional relevante de pacientes oriundos de outros municípios (que não o do próprio hospital), além de valorizar o leito efetivamente ocupado e a efetividade da atenção primária. Tais regras são pactuadas na Comissão Intergestores Bipartite, fórum específico de deliberações do SUS ${ }^{\mathrm{v}}$. Tais regras, recentemente pactuadas, guardam coerência com a diretriz de descentralização, delineada pelo Plano Diretor de Regionalização (PDR-SUS/MG).

\subsection{O Plano Diretor de Regionalização de Minas Gerais como modelo de Descentralização do SUS}

O Sistema Único de Saúde (SUS) foi criado pela Constituição de 1988 com uma rede regionalizada e hierarquizada de ações e serviços de saúde:

Art. 198. As ações e serviços públicos de saúde integram uma rede regionalizada e hierarquizada e constituem um sistema único, organizado de acordo com as seguintes diretrizes:

I - descentralização, com direção única em cada esfera de governo;

II - atendimento integral, com prioridade para as atividades preventivas, sem prejuízos dos serviços assistenciais;

III - participação da comunidade (BRASIL, 1988 - grifos nossos)

A hierarquização ocorre no âmbito dos procedimentos e dos prestadores. O Decreto Federal $n^{\circ}$. 7508, de 2011, que regulamentou a Lei 8080, em seu artigo $8^{\text {a }}$, dispõe que o "acesso universal, igualitário e ordenado às ações e serviços de saúde se inicia pelas Portas de Entrada do SUS e se completa na rede regionalizada e hierarquizada, de acordo com a complexidade do serviço" (BRASIL, 2012, grifos nossos).

Assim, as consultas, exames, cirurgias e demais procedimentos são organizados em níveis de atenção (ou de complexidade tecnológica) nas categorias de atenção primária, média e alta complexidades. Tal classificação, no plano normativo, origina-se de portarias do Ministério da Saúde ${ }^{\mathrm{vi}}$. Já os prestadores podem ser hierarquizados em unidades primárias (postos de saúde, ambulatórios), secundárias (policlínicas, hospitais simples) e terciárias (hospitais gerais e especializados), dependendo da gama e volume de procedimentos que 
ofertarem. Um prestador de nível hierárquico mais complexo (um hospital), irá acumular, consequentemente, procedimentos inerentes aos níveis de complexidade tecnológica inferiores.

Tanto Mendes e Pestana (2004) como La Forgia e Couttolenc (2008, op. cit) consideram que os municípios, embora tenham arregimentado uma autonomia excessiva na década de 90, padecem com a falta de coordenação e capacidade técnica limitada de organizarem seus prestadores pelos níveis de atenção. Akhavan (2001) caracterizou a descentralização como "choque de sistema", pois "as instâncias subnacionais estavam despreparadas para absorver responsabilidades pela prestação de serviços de saúde" (Akhavan, 2001, apud La Forgia e Couttolenc, 2008). Para fazer frente a esses problemas, destaca-se que

A regulamentação adotada em 2006 [Portaria MS/GM no 399, que estabeleceu o Pacto Pela Saúde] representou um avanço, porque fortaleceu a estratégia de regionalização e definiu o papel dos estados, aumentando suas responsabilidades em um sistema regional em desenvolvimento. (La Forgia e Couttolenc, 2008, p. 22)

Assim, na dinâmica de um sistema público de serviços de saúde, os territórios são, também, espaços de responsabilização sanitária. É necessário que haja uma definição das ações e dos serviços de saúde que cada território ofertará a grupos populacionais adscritos (Mendes e Pestana, 2004). Assim, uma dentre as questões a serem avaliadas e planejadas numa região, é o cumprimento de seu papel, isoladamente e na relação com os demais níveis hierárquicos de complexidade. Retomando e complementando Pestana e Mendes, a cada nível de atenção está atrelado um rol de responsabilidades, de funções, a serem operacionalizadas pelas unidades prestadoras de serviços localizadas em cada território ou região de saúde ${ }^{\text {vii }}$.

O Plano Diretor de Regionalização (PDR) é um dos instrumentos de planejamento do SUS, introduzido inicialmente pela Portaria MS/GM No. 399/2006. Através dele, tem-se que uma região é um conjunto de municípios periféricos a um pólo, sendo que este último exerce força de atração sobre os demais. (MINAS GERAIS, 2010)

O PDR estabelece a regionalização hierárquica da assistência à saúde em cada estado da federação, com o objetivo de direcionar a descentralização da rede de serviços para promover o acesso dos usuários aos serviços, considerando os princípios da equidade, integralidade e economia de escala (MINAS GERAIS, 2010). A regionalização da assistência no âmbito do SUS é hierarquizada de acordo com a seguinte lógica: os municípios são agrupados em regiões (microrregiões), que, por sua vez, compõem as regiões ampliadas (macrorregiões) de saúde. O PDR em vigência em Minas Gerais ${ }^{\text {viii }}$ divide o estado em 77 regiões de saúde e 13 regiões ampliadas de saúde.

Ainda de acordo com o PDR, a cada nível de atenção deve corresponder um nível de regionalização. Assim, o conjunto de serviços hospitalares esperados para o nível municipal compõe um conjunto de procedimentos da atenção hospitalar básica. Embora tenha uma menor diversidade de procedimentos componentes, tal nível de agregação apresenta a maior freqüência de ocorrência dentre todos os níveis.

A atenção secundária é ofertada em hospitais e ambulatórios que prestam assistência nas especialidades básicas (pediátrica, clínica médica), serviços de urgência e emergência. São organizados no âmbito das regiões, que são áreas de planejamento de saúde responsáveis pela oferta desses serviços.

Já a atenção de terceiro nível é integrada pelos serviços especializados, organizados em hospitais mais complexos (geralmente, sediados em municípios-polo de regiões ampliadas de saúde). Abrangem procedimentos de maior custo, já que requerem equipamentos de alta 
tecnologia e recursos humanos altamente especializados - normalmente, escassos no mercado. A frequiência de ocorrência de procedimentos desse nível é inferior aos demais, o que demonstra uma necessidade de sua concentração em determinados centros. Ocorrem neste nível, cirurgias neurológicas (LÉLIS, 2012).

Em cada região deve haver um pólo, cujas unidades assistenciais devem responder por funções específicas na organização de serviços que requerem referência e cobertura intermunicipal ou interregional. Portanto, a cada nível de atenção deve corresponder um nível de regionalização.

Como os procedimentos mais complexos apresentam elevados custos fixos, a economia de escala é fundamental para uma alocação eficiente de recursos. Dessa forma, a regionalização da assistência à saúde é um meio de promover a eficiência na aplicação dos recursos investidos e ampliar o acesso a serviços, uma vez que permite a centralização destes em alguns municípios polos de atendimento.

Nesse ínterim, um relatório de 2006 da Organização Mundial de Saúde (OMS) apontou, no Brasil, uma concentração de médicos e outros profissionais da saúde nos grandes centros urbanos, que teriam unidades hospitalares de melhor performance. Segundo a OMS, $a$ "presença de hospitais escola nas grandes cidades teria a capacidade de treinar e atrair profissionais de saúde, causando a formação de centros modernos com menores necessidades e maiores recursos" (GONÇALVES et. al, 2009).Corroborando a influência da economia de escala para o desempenho hospitalar, La Forgia e Couttolenc (2008, op. cit) concluíram que hospitais de menor porte possuem eficiência menor que hospitais de grande porte. (LA FORGIA e COUTTOLENC, 2008, apud GONÇALVES et. al, 2009).

A função, no caso das unidades hospitalares distribuídas nos pólos e regiões, resulta da incorporação de tecnologias gerenciais e assistenciais. O que se procura, no momento inicial de planejamento da distribuição espacial dos serviços de saúde aos prestadores - atuais e potenciais -, é dimensionar as necessidades mais gerais de cada nível regional: o municipal ou local, o micro ou o macrorregional, sempre tendo a economia de escala como princípio norteador. A integralidade e o acesso se tornam possíveis mediante a integração de diversos pontos de atenção dos diferentes níveis de regionalização.

O dimensionamento da capacidade atual e ideal dos territórios deve passar por uma parametrização, para que o planejamento da distribuição de serviços, e sua consequiente avaliação, possam ter uma referência comparável. Daqui se destaca, como aspecto do planejamento, a tipologia de serviços.

\section{METODOLOGIA}

Viu-se, no Referencial Teórico, algumas das dimensões que podem influenciar o desempenho de unidades hospitalares. Nesse trabalho, analisa-se o desempenho das regiões que sediam os hospitais a partir do que se espera de cada um deles, a partir de um modelo de regionalização, expresso nos princípios e diretrizes operacionais do PDR-SUS/MG.

Tais diretrizes tomam corpo na Tipologia ou Carteira de Serviços Hospitalares, que se constitui de um reagrupamento dos procedimentos da tabela do Sistema de Informações Hospitalares (SIH) do Ministério da Saúde. Tal Carteira se agrupa por nível de atenção e tem o objetivo de estabelecer referências para a descentralização e avaliação dos serviços de saúde.

Para a elaboração do documento (Carteira) que contém a Tipologia, num primeiro momento, toma-se a relação dos procedimentos hospitalares que compõem uma Autorização de Internação Hospitalar (AIH), a partir da mesma classificação utilizada pelo Ministério da Saúde, que prevê o seguinte ordenamento, tal qual uma rubrica orçamentária: grupo, subgrupo, forma de organização e procedimento. 
A partir dessa relação, apura-se a frequiência de ocorrência de cada um desses procedimentos, através do aplicativo TABWIN/DATASUS, por município de atendimento. Do resultado dessa frequiência, é possível realizar hipóteses preliminares sobre a possibilidade de descentralização de tais procedimentos, na medida em que se verifica sua concentração ou dispersão pelos territórios (i. e. diferentes níveis de regionalização). Se determinado procedimento está constando numa freqüência considerável em vários municípios que não sejam sede de regiões, por exemplo, é um indicativo que a sua escala não seja significativa para justificar sua concentração em grandes pólos de atendimento.

Num segundo momento, os registros de frequiência da ocorrência dos procedimentos são agrupados por especialidade (cardiologia, oncologia, ortopedia, urologia, neurologia, dentre outros), visando permitir a análise por especialistas de cada uma dessas áreas.

Para essa análise, os levantamentos quantitativos dos procedimentos de cada especialidade são apresentados a todos os especialistas presentes numa reunião presencial. Mediante explanação do levantamento, um coordenador inquire dos especialistas, para cada um dos procedimentos avaliados, se há possibilidade de descentralizá-lo ou se seria necessário concentrá-lo. Isso a partir dos requisitos mínimos que cada prestador deve possuir para poder realizá-lo com qualidade, dada uma suficiência de escala.

Para a obtenção do consenso dos especialistas, aplica-se o método Delphi modificado, cujo objetivo é conseguir uma opinião coletiva sobre determinadas questões, através de uma técnica de estruturação de um processo de comunicação colegiada, que permite a um grupo de indivíduos tratar de questões complexas (PIOLA et. al, 2002, p. 181).

Vencidas essas etapas, tem-se uma lista final de grupos de procedimentos por grupos de especialidades. Tais especialidades são classificadas por níveis de atenção (básico, secundário ou terciário). Essa agregação não coincide, necessariamente, com a classificação utilizada pela tabela de procedimentos do Ministério da Saúde (SIGTAP). A tomografia, por exemplo, embora seja considerado um exame de "alta complexidade" pelo SIGTAP, está classificada pelo PDR-SUS/MG como procedimento da atenção secundária, em vez de atenção terciária.

Significa dizer que trata-se de um exame passível de ser realizado num município que não seja necessariamente sede de uma região ampliada de saúde, na qual devem se concentrar os serviços da atenção terciária. Zucchi et. Al (2000) elencaram o progresso técnico-médico e a difusão da inovação como fatores que agem na expansão da oferta da assistência à saúde e, portanto, na possibilidade de dispersão de determinados serviços mediante redução da escala, caso esse da tomografia computadorizada.

Em contraste, La Forgia e Couttolenc (2008, op. cit) chamam atenção para as distorções na distribuição de equipamentos para tratamentos diagnósticos de alta complexidade. Argumentam que a densidade desses equipamentos é excessivamente alta em alguns municípios, tanto grandes quanto pequenos, mas é quase inexistente na maior parte do país, especialmente da tomografia computadorizada e da ressonância magnética. ${ }^{\mathrm{ix}}$ (La Forgia e Couttolenc, 2008, p. 35).

Enfim, a organização da oferta de serviços, com base na Carteira de Serviços Hospitalares, mostra uma preocupação com a sustentabilidade dos serviços, e, consequentemente, com a garantia do acesso oportuno e qualificado, uma vez que procedimentos menos demandados, e de custo fixo mais elevado, devem ser concentrados em determinados municípios. Lélis (2012) salienta que, ao se buscar a maximização da utilização dos recursos disponíveis, é possível que se atenda "um maior número de pessoas e amplie a acessibilidade assistência à saúde, favorecendo a fixação de profissionais e desenvolvimento de suas habilidades, o que, por sua vez, aumenta a qualidade dos serviços prestados" (LÉLIS, 2012, p. 12). 
A partir da Tipologia, a avaliação da regionalização é realizada por meio da Resolubilidade da Assistência Hospitalar. Esse é um dos indicadores finalísticos da SES/MG, pactuado no Plano Plurianual de Ação Governamental (PPAG). Sua mensuração traduz o grau de alcance de dois objetivos prioritários de governo: i) reduzir as disparidades regionais no atendimento à saúde; ii) consolidar as redes de atenção à saúde em todo o Estado. (MINAS GERAIS, 2013, p. 7).

Ele pode ser calculado por nível de atenção, a depender do lócus da análise (nível de regionalização). Para o PPAG, calcula-se a Resolubilidade do nível terciário (macroregional), justamente por ser aquele com um grau de agregação de tecnologia - e, portanto, escala mais complexo. Portanto, exprime o mais alto grau de suficiência de atendimento de uma rede de prestadores de serviços de saúde pública em um dado território. Sua fórmula de cálculo se expressa, em termos percentuais, pela multiplicação da razão abaixo por 100:

Total de procedimentos realizados por residentes da região ampliada de saúde (macrorregião) na própria região ampliada de residência no nível terciário da atenção

Total de procedimentos realizados por residentes da região ampliada de saúde no nível terciário

\section{RESULTADOS E DISCUSSÃO}

A Tabela abaixo evidencia o desempenho das Regiões Ampliadas de Saúde, ao longo dos seus dez anos de mensuração:

Tabela1 - Comparativo da Resolubilidade Hospitalar da Atenção de Nível Terciário por Região Ampliada de Saúde - Minas Gerais, 2003 a 2012

\begin{tabular}{|c|c|c|c|c|c|c|c|c|c|c|}
\hline \multirow[b]{2}{*}{ REG GĨO AMP L IADA } & \multicolumn{10}{|c|}{ ANO } \\
\hline & 2003 & 2004 & 2005 & 2006 & 2007 & 2008 & 2009 & 2010 & 2011 & 2012* \\
\hline CENTRO & 99,84 & 99,69 & 99,73 & 99,77 & 99,74 & 99,73 & 99,65 & 99,65 & 99,64 & 99,54 \\
\hline TRIÂNGULO DO SUL & 97,04 & 96 & 97,66 & 98 & 95 & 97,39 & 97,57 & 97,02 & 97,73 & 97,14 \\
\hline TRIÂNGULO DO NORTE & 94,1 & 95,75 & 98,02 & 97,76 & 97,57 & 96,33 & 97,21 & 96,28 & 95,66 & 95,06 \\
\hline SUDESTE & 95,52 & 96,14 & 95,73 & 96,35 & 96,72 & 97,02 & 96,12 & 95,49 & 95,43 & 95,91 \\
\hline NORTE & 89,87 & 92,9 & 92 & 93 & 93 & 92,35 & 93,59 & 92,55 & 93,89 & 94,02 \\
\hline SUL & 90,42 & 91 & 90 & 91 & 92 & 91,38 & 90,89 & 91,72 & 92,84 & 93,87 \\
\hline LESTE & 63,08 & 67 & 62,94 & 70 & 69 & 74,65 & 74,54 & 75,25 & 75,85 & 76,33 \\
\hline NOROESTE & 51 & 53,15 & 53,07 & 48,96 & 48,39 & 29,21 & 51,66 & 56,48 & 63,62 & 67,33 \\
\hline CENTRO-SUL & 44 & 51 & 52 & 55 & 58 & 60,96 & 56,23 & 59 & 56,34 & 55,01 \\
\hline OESTE & 51,79 & 55,76 & 51,9 & 48,52 & 47,76 & 46,72 & 48,51 & 48 & 55,96 & 55,05 \\
\hline NORDESTE & 59,32 & 59,55 & 56,07 & 55,05 & 46,63 & 37,24 & 41,73 & 42,34 & 53,16 & 56,94 \\
\hline LESTE DO SUL & 49 & 52,86 & 56,33 & 49,71 & 46 & 30,04 & 32,55 & 45,25 & 48,66 & 49,9 \\
\hline JEQUITINHONHA & 33,5 & 24,96 & 21,14 & 25,66 & 29,72 & 20,82 & 22,59 & 23,93 & 24,85 & 27,53 \\
\hline
\end{tabular}

Fonte: SES/MG; DATASUS; consulta em junho de 2012

*Nota: Dados sujeitos à revisão, devido ao prazo-limite de 6 meses de reapresentação das AIHs.

Percebe-se que, no período avaliado, dez, dentre as treze regiões, apresentaram ampliação de sua resolubilidade. O impacto nesse indicador decorre, fundamentalmente, da expansão da oferta de serviços. O maior aumento ocorreu na Região Ampliada (RA) Noroeste, possivelmente em função do credenciamento do serviço de oncologia em seu município-sede, Patos de Minas.

No entanto, nem sempre a expansão do atendimento à clínica oncológica é suficiente para impactar o cálculo geral da resolubilidade, caso da RA Sul. Provavelmente, pelo volume da demanda.

As Macros Nordeste, Jequitinhonha apresentaram queda. Na Leste do Sul, houve manutenção de um percentual próximo do inicial. Apesar de localizadas em áreas socioeconomicamente favorecidas, as RAs Oeste, Centro-Sul e Leste do Sul registram resolubilidades ainda medianas.

Alguns elementos que apontam para as razões tanto da queda, quanto pelos baixos números, são a baixa escala populacional e a dificuldade de fixação de equipes multiprofissionais de grande especialização. 
Já no caso da RA Oeste, as razões acima elencadas não se aplicam, mas sim um reconhecido déficit de capacidade instalada. Além da incipiência da oferta de leitos de terapia intensiva em todo o território dessa RA, somente um hospital possui Unidades de Terapia Intensiva infantil e neonatal. Portanto, seus pacientes residentes demandam transferências para outras RÃS com freqüência.

Quanto à RA Centro, nota-se uma estabilização acima de 99\%. Tal fato sugere que o aumento da resolubilidade da atenção terciária em um patamar tão elevado requer grande esforço e é de difícil alcance, uma vez que implica em credenciamento de altíssima especialidade e demanda muito rara.

\section{CONSIDERAÇÕES FINAIS}

A análise do indicador da Resolubilidade da Assistência Hospitalar explicita as deficiências dos territórios sanitários. Além de avaliar a descentralização da assistência, pode direcionar o desenvolvimento de ações prioritárias que, por sua vez, contribuirão para a redução das desigualdades entre regiões.

As constatações apresentadas refletem a ampliação geral do acesso aos serviços de saúde mais complexos. No entanto, quando se contrastam as regiões com condições sócioeconômicas mais díspares, prevalece a manutenção das iniquidades regionais, como apontado por Galvarro et. al (2012, p. 26)

Estabelecer referências para descentralização dos serviços de saúde favorece, num plano superior, a organização dos níveis de atenção e sua conseqüente distribuição nos espaços regionais. Sobretudo para essa finalidade, o reagrupamento da Tipologia Hospitalar considerou agregação de densidade tecnológica por níveis de atenção e as economias de escala e de escopo.

Ainda que de forma simples, o trabalho mostra a evolução da descentralização dos serviços hospitalares no estado de Minas Gerais, a partir da Resolubilidade da Assistência. O Plano Diretor de Regionalização, ao se utilizar de tais métodos, constitui-se de base populacional e territorial para cálculos do planejamento em saúde.

Essa constatação traz implicações importantes para os gestores dessa área. Uma delas é que o credenciamento de serviços, desprovido de critérios sólidos de planejamento, pode ser desastroso, pois pode não se sustentar ao longo do tempo, em função de demanda rarefeita.

O estudo apresenta como limitações o escopo de indicador - restrito ao nível terciário da atenção hospitalar -, os vieses a que podem se sujeitar as bases de dados do DATASUS/SIH (conforme discutido em GONÇALVEs et. al, 2009, p. 44), a nãodiscriminação dos gastos por região de saúde e outros fatores sócio-econômicos que podem interferir na descentralização dos serviços. A ampliação do escopo, bem como a investigação das hipóteses levantadas como explicações para os resultados são temas sugeridos para pesquisas futuras.

Refinar a medida da resolubilidade, considerando ferramentas estatísticas como análise de clusters, a análise de regressão, ou ainda, a Teoria das Restrições, merece ser objeto de pesquisas vindouras. Correlações poderão indicar, por exemplo, que a restrição se localiza fora de alguns hospitais, por sua baixa escala.

\section{REFERÊNCIAS}

BRASIL. Constituição da República Federativa do Brasil: promulgada em 5 de outubro de 1988. Disponível em 〈http://www.planalto.gov.br/ccivil_03/constituicao/constituicao.htm> 
Decreto 7508, de 28 de junho de 2011. Regulamenta a Lei no 8.080, de 19 de setembro de 1990, para dispor sobre a organização do Sistema Único de Saúde - SUS, o planejamento da saúde, a assistência à saúde e a articulação interfederativa, e dá outras providências. Disponível em <http://www.planalto.gov.br/ccivil_03/_ato20112014/2011/decreto/D7508.htm>

Portaria GM/MS 399, de 22 de fevereiro de 2006. Divulga o Pacto pela Saúde 2006 - Consolidação do SUS e aprova as Diretrizes Operacionais do Referido Pacto. Disponível em <http://bvsms.saude.gov.br/bvs/saudelegis/gm/2006/prt0399_22_02_2006.html>

GALVARRO, M. P. S. Q. S., FARIA, E. R., FERREIRA, M. A. M., SOUZA, G. J. P.,. Desigualdades Regionais na saúde no Estado de Minas Gerais. Revista de Administração Hospitalar e Inovação em Saúde. Belo Horizonte, v.8, nº 8, jan-jul 2012. Disponível em $<$ http://web.face.ufmg.br/face/revista/index.php/rahis/issue/archive $>$. Belo Horizonte: FACE/UFMG e AHMG, 2012.

GONÇALVES, M. Understanding the healthcare system in Great Britain. A comparative study of hospital management in Great Britain and Brazil: cost information use. Birmingham: Aston University Press, 2002 (Thesis capter)

GONÇALVES, M. A., MARANHÃO, R. K. A., SIQUEIRA, P. C. Análise da permanência Hospitalar do SUS para os diagnósticos mais comuns em Minas Gerais, entre Dezembro de 2007 e Dezembro de 2008. Revista de Administração Hospitalar e Inovação em Saúde. Belo Horizonte, v. 2, $\mathrm{n}^{\circ}, \quad 2$, jan-jul 2009. Disponível em <http://web.face.ufmg.br/face/revista/index.php/rahis/issue/archive>. Belo Horizonte: FACE/UFMG e AHMG, 2009.

LA FORGIA, G. M.; COUTTOLENC, B. F. Desempenho Hospitalar no Brasil. São Paulo: Singular, 2009.

LÉLIS, L. J. B. Indicador de Resolubilidade como Instrumento de Avaliação da Regionalização da Assistência à Saúde no SUS/MG. Brasília: CONSAD, 2012

MENDES, E. V., PESTANA, M. V. C. Pacto de Gestão: da Municipalização Autárquica à Regionalização Cooperativa. Belo Horizonte: Secretaria de Estado da Saúde de Minas Gerais, 2004

MINAS GERAIS. Plano Purianual de Ação Governametal. Disponível em: <http://www.planejamento.mg.gov.br> 2013

. Secretaria de Estado da Saúde. A Resolubilidade da Assistência Hospitalar Micro e Macrorregional na Gestão do SUS-MG - 2003/2009. Disponível em: <http://www.saude.mg.gov.br> Belo Horizonte, 2010.

Secretaria de Estado da Saúde. O Plano Diretor de Regionalização da Saúde de Minas Gerais. Disponível em: <http://www.saude.mg.gov.br> Belo Horizonte, 2010.

PIOLA S.F., VIANNA S.M., CONSUELO D.V. Estudo Delphi: atores sociais e tendências do sistema de saúde brasileiro. Cad Saúde Pública 2002; 18(Supl): 181-90. 
OUCHI, W. G. Markets, bureaucracies and clans. Administrative Science Quartely, 25, March, 1980. pp. 129-141.

ZUCCHI, P, DEL NERO, C., MALIK, A.M.Gastos em Saúde: os fatores que agem na demanda e na oferta dos serviços de saúde. Saúde e Sociedade. Pp. 127-150

i Nesse diapasão, podemos citar a dificuldade de se produzir resultados positivos na atenção primária, na medida em que variáveis, como o aumento da adesão a planos de saúde, diminuem o desempenho em indicadores que, aparentemente, não estariam diretamente relacionados a esse nível de atenção da assistência.

ii Em contraste, La Forgia e Coutolenc (2008) alertam que os gastos com saúde no Brasil são altos se comparados com outros países de renda média. Além disso, ponderam que atingir os níveis de gasto per capita do quartil mais baixo dos países da OCDE implicaria aumentar o gasto nacional com saúde três vezes, o que comprometeria cerca de $25 \%$ do PIB nacional com saúde. Esse percentual é muito maior que o de países de alto gasto entre os da OCDE. (LA FORGIA E COUTOLENC, 2008p. 52)

iii O sistema de saúde na Grã-Bretanha (National Health System) evidencia interesse de relevo acerca da transparência dos indicadores de seus prestadores, especialmente os de custo-efetividade. Gonçalves (2002) relatou que, desde 1998, há um relatório publicado anualmente que, com ampla cobertura da imprensa e com avaliação pública, busca induzir o benchmarking entre as unidades.

iv Outro constrangimento para a manutenção de um ambiente colaborativo entre tais organizações é a constatação de que o nível de remuneração da AIH (autorização de internação hospitalar) representa menos da metade do custo real da maioria dos procedimentos hospitalares (LA FORGIA e COUTTOLENC, 2008, p. 22)

${ }^{v}$ Vide Deliberações CIB-SUS/MG 1466 e 1468/2013 - disponíveis em http://www.saude.mg.gov.br

${ }^{v i}$ Disponível para consulta em: http://sigtap.datasus.gov.br/tabela-unificada/app/sec/inicio.jsp

vii La Forgia e Couttolenc obtemperam que "embora algumas experiências estejam em curso, a estruturação de serviços regionalizados e coordenados de saúde ainda precisa se fortalecer no Brasil".(LA FORGIA e COUTTOLENC, 2008, p. 22)

viii Vide Deliberações CIB-SUS/MG 978/2011 e 1219/2012

ix À guisa de exemplo, a região metropolitana do Rio de Janeiro tem 75\% mais aparelhos de TC e RM do que a região metropolitana de Paris, que tem população similar 